\title{
ARTICLE
}

Myelodysplastic syndrome

\section{Toxic iron species in lower-risk myelodysplastic syndrome patients: course of disease and effects on outcome}

\author{
Marlijn Hoeks $\mathbb{1}^{1,2,3} \cdot$ Tim Bagguley $\mathbb{1}^{4} \cdot$ Corine van Marrewijk $\mathbb{1}^{3}$ - Alex Smith ${ }^{4}$ - David Bowen ${ }^{5}$ - Dominic Culligan ${ }^{6}$. \\ Seye Kolade ${ }^{7}$ - Argiris Symeonidis $\mathbb{1}^{8}$. Hege Garelius ${ }^{9} \cdot$ Michail Spanoudakis $^{10,11}$ - Saskia Langemeijer ${ }^{3}$. \\ Rian Roelofs ${ }^{12} \cdot$ Erwin Wiegerinck $^{12} \cdot$ Aurelia Tatic $^{13} \cdot$ Sally Killick ${ }^{14} \cdot$ Panagiotis Panagiotidis $^{15} \cdot$ Oana Stanca $^{16}$. \\ Eva Hellström-Lindberg ${ }^{17} \cdot$ Jaroslav Cermak ${ }^{18} \cdot$ Melanie van der Klauw $^{19} \cdot$ Hanneke Wouters $^{19} \cdot$ Marian van Kraaij $^{3}$. \\ Nicole Blijlevens ${ }^{3} \cdot$ Dorine W. Swinkels $^{12} \cdot$ Theo de Witte $^{20} \cdot$ on behalf of the EUMDS Registry Participants
}

Received: 30 April 2020 / Revised: 3 August 2020 / Accepted: 6 August 2020 / Published online: 18 September 2020

(c) The Author(s) 2020. This article is published with open access

\section{Introduction}

Red blood cell transfusions (RBCT) remain the cornerstone of supportive care in lower-risk myelodysplastic syndrome (LRMDS) [1]. Transfusion dependency in LRMDS patients

Members of the EUMDS Registry Participants are listed below Acknowledgements.

Supplementary information The online version of this article (https:// doi.org/10.1038/s41375-020-01022-2) contains supplementary material, which is available to authorized users.

Marlijn Hoeks

marlijn.hoeks@radboudumc.nl

1 Centre for Clinical Transfusion Research, Sanquin Research, Leiden, The Netherlands

2 Department of Clinical Epidemiology, Leiden University Medical Center, Leiden, The Netherlands

3 Department of Hematology, Radboud University Medical Center, Nijmegen, The Netherlands

4 Epidemiology and Cancer Statistics Group, University of York, York, UK

5 St. James's Institute of Oncology, Leeds Teaching Hospitals, Leeds, UK

6 Department of Hematology, Aberdeen Royal Infirmary, Aberdeen, UK

7 Department of Hematology, Blackpool Victoria Hospital, Blackpool, Lancashire, UK

8 Department of Medicine, Division of Hematology, University of Patras Medical School, Patras, Greece

9 Department of Medicine, Sect. of Hematology and Coagulation, Sahlgrenska University Hospital, Göteborg, Sweden

10 Department of Hematology, Airedale NHS Trust, Airdale, UK

11 Department of Haematology, Warrington and Halton Teaching is associated with inferior outcomes, mainly attributed to severe bone marrow failure [2]. However, iron toxicity, due to frequent RBCT or ineffective erythropoiesis, may be an additional negative prognostic factor [3-6]. Recently, much progress has been made in unraveling the iron metabolism. The peptide hormone hepcidin is the key regulator by inhibiting iron uptake through degradation of ferroportin, a cellular iron exporter [7]. Erythroferrone and GDF15, produced by erythroblasts, inhibit hepcidin production, which leads to increased uptake and cellular release of iron for the purpose of erythropoiesis [8].

Hospitals NHS foundation Trust, Cheshire, UK

12 Department of Laboratory Medicine, Hepcidinanalysis.com, and Radboudumc Expertise Center for Iron Disorders, Radboud University Medical Center, Nijmegen, The Netherlands

13 Center of Hematology and Bone Marrow Transplantation, Fundeni Clinical Institute, Bucharest, Romania

14 Department of Hematology, Royal Bournemouth Hospital, Bournemouth, UK

15 Department of Haematology, 1st Department of Propedeutic Internal Medicine, National and Kapodistrian University of Athens, Medical School, Laikon General Hospital, Athens, Greece

16 Department of Hematology, Coltea Clinical Hospital, Bucharest, Romania

17 Department of Medicine, Division of Hematology, Karolinska Institutet, Stockholm, Sweden

18 Department of Clinical Hematology, Institute of Hematology and Blood Transfusion, Praha, Czech Republic

19 Department of Endocrinology, University of Groningen, University Medical Center Groningen, Groningen, The Netherlands

20 Nijmegen Center for Molecular Life Sciences, Department of Tumor Immunology, Radboud University Medical Center, Nijmegen, The Netherlands 
The pathophysiology of iron metabolism in MDS is still not completely understood. Exceedingly high reactive oxygen species (ROS) levels are associated with iron toxicity, disease development, and progression in MDS patients [9-12]. Malondialdehyde (MDA), resulting from lipid peroxidation of polyunsaturated fatty acids, is a biomarker of oxidative stress [10,12]. Currently, little is known about the prognostic impact of ROS in MDS patients.

The aim of this study is twofold: (1) describe iron and oxidative stress parameters over time in LRMDS patients and (2) to assess their effect on overall and progression-free survival.

\section{Materials and methods}

The EUMDS registry prospectively collects observational data on newly diagnosed LRMDS patients from 148 centers in 16 countries in Europe and Israel as of January 2008. All patients provided informed consent. Clinical data were collected at baseline and at each six-monthly follow-up visit. Serum samples were collected prospectively at each visit from 256 patients included in six participating countries. Conventional iron parameters were measured with routine assays. We additionally analyzed hepcidin, growth differentiation factor 15 (GDF15), soluble transferrin receptor (sTfR), nontransferrin bound iron (NTBI), labile plasma iron (LPI), and MDA. Subjects were prospectively followed until death, loss to follow-up, or withdrawal of consent.

All iron parameters were measured centrally at the department of Laboratory Medicine of the Radboudumc, Nijmegen, The Netherlands. Serum samples were collected just prior to transfusion in transfusion-dependent patients and stored at $-80^{\circ} \mathrm{C}$. Details on the assays and reference ranges of hepcidin, GDF15, sTfR, NTBI, LPI, and MDA are provided in the supplement.

The Spearman rank test was used to evaluate correlations between iron parameters. We stratified the results by transfusion dependency per visit and the presence of ring sideroblasts. When evaluating temporal changes in iron parameters, with linear quantile mixed models, we excluded patients from the timepoint they received iron chelation therapy. Overall survival (OS) was defined as the time from MDS diagnosis to death or, in case of progression-free survival, to date of progression or death; patients still alive at the end of follow-up were censored. Time-dependent Kaplan-Meier curves and cox proportional hazards models were used.

\section{Results}

In total, 256 consecutive patients, were included in this study. Over five six-monthly visits, 1040 samples were
Table 1 Baseline characteristics.

\begin{tabular}{|c|c|}
\hline & $N(\%)$ \\
\hline Total & $256(100.0)$ \\
\hline \multicolumn{2}{|l|}{ Sex } \\
\hline Males & $169(66.0)$ \\
\hline Females & $87(34.0)$ \\
\hline \multicolumn{2}{|l|}{ Age } \\
\hline $35-44$ & $2(0.8)$ \\
\hline $45-54$ & $7(2.7)$ \\
\hline $55-64$ & $51(19.9)$ \\
\hline $65-74$ & $78(30.5)$ \\
\hline $75+$ & $118(46.1)$ \\
\hline Mean (sd) & $72.1(9.5)$ \\
\hline Median (min-max) & $74.0(37.0-95.0)$ \\
\hline \multicolumn{2}{|l|}{ MDS diagnosis } \\
\hline RCMD & $114(44.5)$ \\
\hline RARS & $56(21.9)$ \\
\hline RA & $45(17.6)$ \\
\hline RAEB-1 & $16(6.3)$ \\
\hline RCMD-RS & $10(3.9)$ \\
\hline 5q-syndrome & $10(3.9)$ \\
\hline MDS-U & $5(2.0)$ \\
\hline \multicolumn{2}{|l|}{ Group } \\
\hline NonRS-TI & $143(55.9)$ \\
\hline NonRS-TD & 47 (18.4) \\
\hline RS-TI & $48(18.8)$ \\
\hline RS-TD & $18(7.0)$ \\
\hline \multicolumn{2}{|l|}{ IPSS-R category } \\
\hline Very low/low & $195(76.2)$ \\
\hline Intermediate & $23(9.0)$ \\
\hline High/very high & $4(1.6)$ \\
\hline Not known & $34(13.3)$ \\
\hline \multicolumn{2}{|l|}{ IPSS category } \\
\hline Low risk & $144(56.3)$ \\
\hline Intermed-1 & $75(29.3)$ \\
\hline Intermed-2 & $1(0.4)$ \\
\hline Not known & $36(14.1)$ \\
\hline \multicolumn{2}{|l|}{ Karnofsky performance status } \\
\hline Able to work and normal activity & $193(75.4)$ \\
\hline Unable to work & $48(18.8)$ \\
\hline Unable to care for self & $1(0.4)$ \\
\hline Not known & $14(5.5)$ \\
\hline \multicolumn{2}{|l|}{ Comorbidity index } \\
\hline Low risk & $158(61.7)$ \\
\hline Intermediate risk & 79 (30.9) \\
\hline High risk & $19(7.4)$ \\
\hline \multicolumn{2}{|l|}{ EQ5D index score } \\
\hline Mean (sd) & $0.77(0.24)$ \\
\hline Median (p10-p90) & $0.80(0.52-1.00)$ \\
\hline
\end{tabular}


Table 1 (continued)

\begin{tabular}{lc}
\hline & $N(\%)$ \\
\hline ESA & $159(62.1)$ \\
$\quad$ No & $97(37.9)$ \\
$\quad$ Yes & $241(94.1)$ \\
Iron chelation & $15(5.9)$ \\
$\quad$ No & $5(2.0)$ \\
Yes & $11(4.3)$ \\
$\quad$ Desferoxamine & \\
$\quad$ Deferiprone/deferasirox & $245(95.7)$ \\
Hypomethylating agents & $11(4.3)$ \\
No & \\
Yes & $4.8(3.9-$ not reached) \\
Overall survival & \\
Median (95\% CI) & $15(34.1)$ \\
Cause of death & $24(54.5)$ \\
MDS unrelated & $5(11.4)$ \\
MDS related & \\
Unknown & $6.6(5.9-7.0)$ \\
Follow-up time (censored last EUMDS visit) & \\
Median (95\% CI) & \\
\hline
\end{tabular}

sd standard deviation, $M D S$ myelodysplastic syndrome, RCMD refractory cytopenia with multilineage dysplasia, $R A R S$ refractory anemia with ring sideroblasts, $R A$ refractory anemia, $R A E B$ refractory anemia with excess blasts, $R C M D-R S$ refractory cytopenia with multilineage dysplasia with ring sideroblasts, $M D S-U$ myelodysplastic syndrome unspecified, $R S$ ring sideroblasts, $T I$ transfusion-independent, $T D$ transfusion-dependent, IPSS(-R) (revised) international prognostic scoring system, EQ5D EuroQoL five dimension scale, $E S A$ erythroid stimulating agents.

collected. Table 1 describes the patient characteristics. Most patients without ring sideroblasts were transfusionindependent at diagnosis (nonRS-TI; 55.9\%), 18.8\% with ring sideroblasts were transfusion-independent (RS-TI), $18.4 \%$ without ring sideroblasts were transfusion-dependent (nonRS-TD), and 7\% with ring sideroblasts were transfusion-dependent patients (RS-TD). The median follow-up time was 6.6 years (95\% CI 5.9-7.0).

LPI was positively correlated with transferrin saturation (TSAT) $(r=0.15, p<0.001$, Fig. S1). LPI values increased exponentially at TSAT values above $80 \%$. This effect was most pronounced in the transfusion-dependent groups, but also observed in the RS-TI group. MDA was weakly correlated with NTBI $(r=0.09, p=0.069)$ and negatively correlated with hemoglobin level $(r=-0.1, p=0.033)$. GDF15 and hepcidin were negatively correlated in the RSTI and nonRS-TD group and significantly negatively correlated in the RS-TD group $(r=-0.34, p=0.007$, Fig. S2).

Serum ferritin levels were elevated in all subgroups with a mean value of $858 \mu \mathrm{g} / \mathrm{L}$ at visit 5 . The highest serum ferritin levels were observed in the RS-TD group (mean value at visit 5: $2092 \mu \mathrm{g} / \mathrm{L}$, Table S1). Serum ferritin increased significantly per visit in the RS-TD group (beta $454.46 \mu \mathrm{g} / \mathrm{L} ; 95 \%$ CI 334.65-574.27), but not in the other groups (Table S2).

All subgroups, except for the nonRS-TI, had elevated TSAT levels. TSAT levels were most markedly increased in the RS-TD group with a mean TSAT of $88 \%$ at visit 5 (Table S1). In both transfusion-dependent groups the median increase per visit was significant (Table S2).

LPI was elevated in the RS-TD group exclusively with a mean value of $0.59 \mu \mathrm{mol} / \mathrm{L}$ at visit 5 (Table $\mathrm{S} 1$ ). NTBI was elevated in all subgroups, with the highest values in the RSTD group (Table S1). The increase in median NTBI level was significant in both transfusion-dependent groups (Table S2).

Hepcidin levels were markedly elevated in the nonRSTD group. Interestingly, hepcidin levels were lower in the RS-TD group, probably reflecting ineffective erythropoiesis, likewise supported by lower hepcidin/ferritin ratios in RS groups (Table S1). Median hepcidin levels increased over time in the transfusion-dependent subgroups only (Table S2).

GDF15 levels, analyzed in the light of its potential role in hepcidin suppression, were increased in all subgroups (Table S1). The RS subgroups had higher GDF15 levels compared to the nonRS groups, reflecting increased erythropoiesis.

Mean sTfR levels were within the reference range in all subgroups except for the RS-TI group, which showed elevated levels, reflecting increased erythropoiesis (Table S1).

MDA levels were within the reference range in the nonRS-TI group and above the upper limit of the reference range in all other subgroups with the highest levels in the RS-TD group (Table S1). MDA levels at diagnosis were markedly higher in the RCMD-RS group compared to other subtypes (Table S3.1). As expected, in the group with elevated MDA levels, the transfusion density was markedly higher as compared with patients with low MDA levels (Table S3.2). Overall MDA levels increased over time ( $p<$ $0.0001)$. The steepest increase was observed in transfusiondependent patients, with the highest median levels over time in the RS-TD group (Table S3.3).

\section{Overall survival (OS)}

Figure 1 shows a Kaplan-Meier curve for OS, stratified by LPI above or below the lower limit of detection (LLOD) and transfusion status as time-varying variables. Transfusion-dependent patients with elevated LPI levels have inferior OS compared to other subgroups. The Cox model shows an adjusted hazard ratio (HR) for OS, corrected for age at diagnosis and IPSS-R, of 2.7 (95\% CI $1.5-5.0, p=0.001)$ for LPI $>$ LLOD. With the transfusion- 


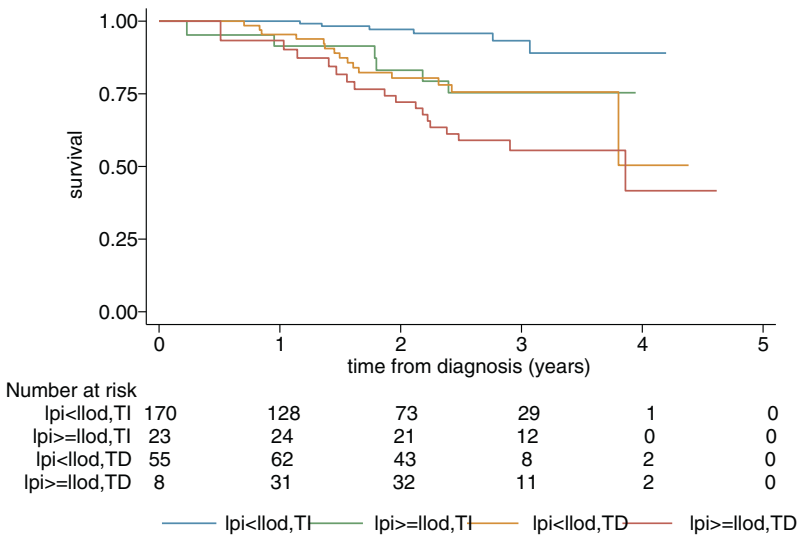

Fig. 1 Kaplan-Meier curve overall survival stratified by labile plasma iron above or below the lower limit of detection and transfusion status as time-dependent variables. LPI labile plasma iron, LLOD lower limit of detection, TI transfusion-independent, TD transfusion-dependent.

independent group with LPI values $<$ LLOD as a reference, the HR for OS in the transfusion-independent group with LPI > LLOD was 4.5 (95\% CI 1.4-13.9, $p=0.01$ ), for the transfusion-dependent group with LPI <LLOD: 3.9 (95\% CI 1.5-10.4, $p=0.006$ ), and for the transfusion-dependent group with LPI > LLOD: 6.7 (95\% CI 2.5-17.6, $p<0.001$, Table S4).

The adjusted HR for OS for elevated NTBI was $1.6(95 \%$ CI $0.8-3.1, p=0.17$ ). Transfusion-independent patients with normal NTBI levels have superior OS when compared with the other subgroups, who have significantly increased HRs for OS (Table S5).

Elevated TSAT $(>80 \%)$ alone did not influence OS. However, when we repeated the analysis in the whole EUMDS registry as a dichotomous and continuous variable ( $n=1076,2853$ visits), elevated TSAT did influence OS with an adjusted HR of 2.1 (95\% CI 1.6-2.7, $p<0.001)$ and 1.009 (95\% CI 1.004-1.014, $p<0.001$ ), respectively. Transfusion-dependent patients with a TSAT $\geq 80 \%$ had the worst OS with an adjusted HR of 4.2 (95\% CI 2.9-5.9, $p<$ $0.001)$.

\section{Progression-free survival}

In line with the effect of LPI on OS progression-free survival is significantly inferior in transfusion-dependent patients with LPI levels >LLOD (HR 9.2, 95\% CI $3.8-22.5, p<0.001)$.

\section{Discussion}

The results of this study suggest that LRMDS patients who are transfusion-dependent and have a MDS subtype with ring sideroblasts have the highest levels for markers that reflect iron toxicity. Likewise, the highest hepcidin levels were observed in the transfusion-dependent nonRS group, but importantly, hepcidin levels and hepcidin/ferritin ratios were markedly lower in the transfusion-dependent patients with ring sideroblasts. Despite the excess of iron due to RBCT, hepcidin levels were lower than expected, thereby increasing the iron uptake from the gut and release of iron from the reticulo-endothelial system. Transfusion dependency is a known risk factor for iron toxicity. However, ineffective erythropoiesis in RS subgroups evidently leads to additional iron toxicity and potentially to increased morbidity and mortality [13-15]. Therefore, transfusiondependent LRMDS patients with ring sideroblasts should be closely monitored for signs of iron toxicity and treated accordingly.

Our data suggest that LPI levels above the LLOD are associated with inferior overall and progression-free survival, irrespective of transfusion status. This highlights the importance of rational RBCT strategies in LRMDS patients. Novel hepcidin regulators as erythroferrone, hepcidin agonists, and early start of iron chelation are subjects for future research.

Overall MDA levels, as a marker of oxidative stress, increased significantly over time in our patient group. Oxidative stress due to iron toxicity could lead to organ damage as well as mutagenesis and clonal instability contributing to a higher progression risk [9-12]. Nevertheless, MDA is not an exclusive marker for oxidative stress, future research should focus on both oxidant and antioxidant factors thereby unraveling the exact relation between iron toxicity and oxidative stress.

In conclusion, iron toxicity is associated with inferior survival in LRMDS patients. More restrictive RBCT strategies and pre-emptive iron reducing interventions may prevent or reverse these unwanted effects.

Acknowledgements The authors would like to thank the other EUMDS Steering Committee members, local investigators and their teams (Table S4), and patients for their contribution to the EUMDS Registry; Jan Verhagen for his contribution in the measurement of the iron parameters; Margot Rekers, Karin van der Linden, and Siem Klaver for sample handling; Elise van Pinxten-van Orsouw and Linda van der Landen for data entry of all iron parameters; and Louise de Swart for her contribution to the analyses on the iron parameters.

EUMDS Registry Participants R. Stauder ${ }^{21}$, A. Walder ${ }^{22}$, M. Pfeilstöcker $^{23}$, A. Schoenmetzler-Makrai ${ }^{23}$, S. Burgstaller ${ }^{24}$, J. Thaler ${ }^{24}$, I. Mandac Rogulj ${ }^{25}$, M. Krejci ${ }^{26}$, J. Voglova ${ }^{27}$, P. Rohon ${ }^{28}$, A. Jonasova $^{29}$, J. Cermak ${ }^{30}$, D. Mikulenkova ${ }^{30}$, I. Hochova ${ }^{31}$, P. D. Jensen ${ }^{32}$, M. S. Holm ${ }^{33}$, L. Kjeldsen ${ }^{34}$, I. H. Dufva ${ }^{35}$, H. Vestergaard ${ }^{36}$, D. Re ${ }^{37}$, B. Slama ${ }^{38}$, P. Fenaux ${ }^{39}$, B. Choufi ${ }^{40}$, S. Cheze ${ }^{41}$, D. Klepping ${ }^{42}$, B. Salles $^{42}$, B. de Renzis ${ }^{43}$, L. Willems ${ }^{44}$, D. De Prost ${ }^{45}$, J. Gutnecht ${ }^{46}$, S. Courby $^{47}$, V. Siguret $^{48}$, G. Tertian ${ }^{49}$, L. Pascal ${ }^{50}$, M. Chaury ${ }^{51}$, E. Wattel $^{52}$, A. Guerci ${ }^{53}$, L. Legros ${ }^{54}$, P. Fenaux ${ }^{55}$, R. Itzykson ${ }^{55}$, L. Ades $^{55}$, F. Isnard ${ }^{56}$, L. Sanhes ${ }^{57}$, R. Benramdane ${ }^{58}$, A. Stamatoullas ${ }^{59}$, 
S. Amé ${ }^{60}$, O. Beyne-Rauzy ${ }^{61}$, E. Gyan ${ }^{62}$, U. Platzbecker ${ }^{63}$, C. Badra$\operatorname{kan}^{64}$, U. Germing ${ }^{65}$, M. Lübbert ${ }^{66}$, R. Schlenk ${ }^{67}$, I. Kotsianidis ${ }^{68}$, C. Tsatalas $^{68}$, V. Pappa ${ }^{69}$, A. Galanopoulos ${ }^{70}$, E. Michali ${ }^{70}$, P. Panagio-

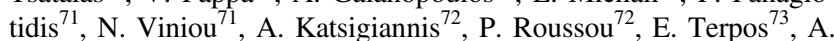
Kostourou $^{74}$, Z. Kartasis ${ }^{75}$, A. Pouli ${ }^{76}$, K. Palla ${ }^{77}$, V. Briasoulis ${ }^{78}$, E. Hatzimichael $^{78}$, G. Vassilopoulos ${ }^{79}$, A. Symeonidis ${ }^{80}$, A. Kourakli ${ }^{80}$, P. Zikos ${ }^{81}$, A. Anagnostopoulos ${ }^{82}$, M. Kotsopoulou ${ }^{83}$, K. Megalakaki $^{83}$, M. Protopapa ${ }^{84}$, E. Vlachaki ${ }^{85}$, P. Konstantinidou ${ }^{86}$, G. Stemer $^{87}$, A. Nemetz ${ }^{88}$, U. Gotwin ${ }^{89}$, O. Cohen ${ }^{89}$, M. Koren ${ }^{89}$, E. Levy $^{90}$, U. Greenbaum ${ }^{90}$, S. Gino-Moor ${ }^{91}$, M. Price ${ }^{92}$, Y. Ofran ${ }^{93}$, A. Winder $^{94}$, N. Goldshmidt ${ }^{95}$, S. Elias, R. Sabag ${ }^{95}$, I. Hellman ${ }^{96}$, M. Ellis $^{96}$, A. Braester ${ }^{97}$, H. Rosenbaum ${ }^{98}$, S. Berdichevsky ${ }^{99}$, G. Itz-

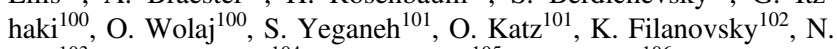
Dali $^{103}$, M. Mittelman ${ }^{104}$, L. Malcovati ${ }^{105}$, L. Fianchi ${ }^{106}$, A. vd Loosdrecht $^{107}$, V. Matthijssen ${ }^{108}$, A. Herbers ${ }^{109}$, H. Pruijt ${ }^{109}$, N. Aboosy ${ }^{110}$, F. de Vries ${ }^{110}$, G. Velders ${ }^{11}$, E. Jacobs ${ }^{112}$, S. Langemeijer ${ }^{113}$, M. MacKenzie $^{113}$, C. Lensen ${ }^{114}$, P. Kuijper ${ }^{115}$, K. Madry ${ }^{116}$, M. Camara $^{117}$, A. Almeida ${ }^{117}$, G. Vulkan ${ }^{118}$, O. Stanca Ciocan ${ }^{119}$, A. Tatic $^{120}$, A. Savic ${ }^{121}$, C. Pedro ${ }^{122}$, B. Xicoy ${ }^{123}$, P. Leiva ${ }^{124}$, J. Munoz $^{125}$, V. Betés ${ }^{126}$, C. Benavente ${ }^{127}$, M. Lozano ${ }^{128}$, M. Martinez $^{128}$, P. Iniesta ${ }^{129}$, T. Bernal ${ }^{130}$, M. Diez Campelo ${ }^{131}$, D. Tormo ${ }^{132}$, R. Andreu Lapiedra ${ }^{133}$, G. Sanz ${ }^{134}$, E. Hesse Sundin ${ }^{135}$, H. Garelius ${ }^{136}$, C. Karlsson ${ }^{137}$, P. Antunovic ${ }^{138}$, A. Jönsson ${ }^{138}$, L. Brandefors ${ }^{139}$, L. Nilsson $^{140}$, P. Kozlowski ${ }^{141}$, E. Hellstrom-Lindberg ${ }^{142}$, M. Grövdal ${ }^{143}$, K. Larsson ${ }^{144}$, J. Wallvik ${ }^{144}$, F. Lorenz ${ }^{145}$, E. Ejerblad ${ }^{146}$, D. Culligan $^{147}$, C. Craddock ${ }^{148}$, S. Kolade ${ }^{149}$, P. Cahalin ${ }^{149}$, S. Killick ${ }^{150}$, S. Ackroyd $^{151}$, C. Wong ${ }^{152}$, A. Warren ${ }^{152}$, M. Drummond ${ }^{153}$, C. Hall ${ }^{154}$, K. Rothwell ${ }^{155}$, S. Green ${ }^{156}$, S. Ali ${ }^{156}$, D. Bowen ${ }^{157}$, M. Karakantza ${ }^{157}$, M. Dennis ${ }^{158}$, G. Jones ${ }^{159}$, J. Parker ${ }^{160}$, A. Bowen ${ }^{160}$, R. Radia ${ }^{161}$, E. Das-Gupta $^{161}$, P. Vyas ${ }^{162}$, E. Nga ${ }^{163}$, D. Creagh ${ }^{164}$, J. Ashcroft ${ }^{165}$, J. Mills $^{166}$, L. Bond ${ }^{167}$

${ }^{21}$ Medical University of Innsbruck, Innsbruck, Austria; ${ }^{22}$ Bezirkskrankenhaus, Lienz, Austria; ${ }^{23}$ Hanusch Krankenhaus, Vienna, Austria; ${ }^{24}$ Klinikum Kreuzschwestern, Wels, Austria; ${ }^{25}$ Clinical Hospital Merkur, Zagreb, Croatia; ${ }^{26}$ The University Hospital Brno, Brno, Czech Republic; ${ }^{27}$ Charles University Faculty of Medicine, Hradec Kralove, Czech Republic; ${ }^{28}$ University Hospital, Olomouc, Czech Republic; ${ }^{29}$ General University Hospital, 1st Clinic of Internal Medicine, Prague, Czech Republic; ${ }^{30}$ General University Hospital, Institute of Hematology and Blood Transfusion, Prague, Czech Republic; ${ }^{31}$ University Hospital Motol, Prague, Czech Republic; ${ }^{32}$ University Hospital, Aalborg, Denmark; ${ }^{33}$ University Hospital, Aarhus, Denmark; ${ }^{34}$ University Hospital: Rigshospitalet, Copenhagen, Denmark; ${ }^{35} \mathrm{Herlev}$ Hospital, Herlev Ringvej, Herlev, Denmark; ${ }^{36}$ Odense University Hospital, Odense, Denmark; ${ }^{37}$ Hospital Center D'antibes Juan-Les-Pins, Antibes, France; ${ }^{38}$ Centre Hospital, Avignon, France; ${ }^{39}$ Hospital Avicenne, Bobigny, France; ${ }^{40}$ Centre Hospital Boulogne-sur-Mer, Boulogne-sur-Mer, France; ${ }^{41} \mathrm{Cen}-$ tre Hospital Universitaire Clemenceau, Caen, France; ${ }^{42}$ Centre Hospital William Morey, Chalon-sur-Saone, France; ${ }^{43}$ Centre Hospital Universitaire, Clermont-Ferrand, France; ${ }^{44}$ Hospital Hotel Dieu, Cochin, France; ${ }^{45}$ Louis-Mourier Hospital, Colombes, France; ${ }^{46} \mathrm{CHI}$ Frejus Saint Raphael, Frejus, France; ${ }^{47} \mathrm{CHU}$ Albert Michallon, Grenoble, France; ${ }^{48}$ Hopital Charles-Foix Ap-Hp, Ivry-sur-Seine, France; 49Hospital Bicetre, Le Kremlin-Bicetre, France; ${ }^{50}$ Hospital St Vincent de Paul, Lille, France; ${ }^{51} \mathrm{CHU}$ Limoges Hospital Dupuytren, Limoges, France; ${ }^{52}$ Hospital Edouard Herriot, Lyon, France; ${ }^{53} \mathrm{CHU}$ Nancy: Hospital Brabois (Vandoeuvre Les Nancy), Nancy, France; ${ }^{54} \mathrm{CHU}$ de Nice: Hospital l'Archet, Nice, France; ${ }^{55} \mathrm{Hopital}$ St Louis, Paris, France; ${ }^{56}$ Hospital Saint-Antoine, Paris, France; ${ }^{57}$ Centre Hospital Marechal Joffre, Perpignan, France; ${ }^{58}$ Centre Hospital de Pontoise, Pontoise, France; ${ }^{59} \mathrm{CHU}$ de Rouen: Hospital Charles-Nicolle, Rouen, France; ${ }^{60} \mathrm{CHU}$ Hospital Hautepierre de Strasbourg, Strasbourg, France; ${ }^{61} \mathrm{CHU}$ Toulouse: Hospital Purpan, Toulouse, Toulouse, France; ${ }^{62} \mathrm{CHRU}$ de Tours, Tours, France; ${ }^{63}$ University
Hospital Carl Gustav Carus, Dresden, Germany; ${ }^{64}$ HELIOS: St. Johannes Hospital in Hamborn, Duisburg, Germany; ${ }^{65}$ Heinrich-Heine University Hospital, Dusseldorf, Germany; ${ }^{66}$ University Hospital Freiburg, Freiburg, Germany; ${ }^{67}$ University Hospital Ulm, Ulm, Germany; ${ }^{68}$ Democritus University of Thrace, Alexandroupolis, Greece; ${ }^{69}$ General Hospital Attikon, University of Athens Medical School, Athens, Greece; ${ }^{70}$ General Hospital G. Gennimatas, Athens, Greece; ${ }^{71}$ General Hospital Laikon, University of Athens Medical School, Athens, Greece; ${ }^{72}$ General Hospital Sotiria, University of Athens Medical School, Athens, Greece; ${ }^{73}$ Hellenic 251 Air Force General Hospital, Athens, Greece; ${ }^{74}$ Pammakaristos Hospital, Athens, Greece; ${ }^{75}$ Patission Prefectural General Hospital: Halkida, Athens, Greece; ${ }^{76} \mathrm{St}$. Savvas Oncology Hospital of Athens, Athens, Greece; ${ }^{77}$ General Hospital of Chania, Chania, Greece; ${ }^{78}$ University Hospital of Ioannina, Ioannina, Greece; ${ }^{79}$ University Hospital of Larissa, Larissa, Greece; ${ }^{80}$ General University Hospital of Patras, Patras, Greece; ${ }^{81}$ St. Andreas General Hospital, Patras, Greece; ${ }^{82}$ General Hospital of Thessaloniki George Papanikolaou, Pilea Chortiatis, Greece; ${ }^{83}$ Metaxa Hospital, Piraeus, Greece; ${ }^{84}$ General Hospital of Serres, Serres, Greece; ${ }^{85}$ Hippokration-General Hospital of Thessaloniki, Thessaloniki, Greece; ${ }^{86}$ Theageneio General Hospital, Thessaloniki, Greece; ${ }^{87} \mathrm{HaEmek}$ Medical Center, Afula, Israel; ${ }^{88}$ Barzilai Medical Center, Ashkelon, Israel; ${ }^{89}$ Asaf-Harofe Medical Center, Be'er Ya'akov, Israel; ${ }^{90}$ Soroka Medical Center, Beersheba, Israel; ${ }^{91}$ Bnai Zion Medical Center, Haifa, Israel; ${ }^{92}$ Carmel Medical Center, Haifa, Israel; ${ }^{93}$ Rambam Medical Centre, Haifa, Israel; ${ }^{94}$ Wolfson Medical Center, Holon, Israel; ${ }^{95}$ Hadassah Medical Center, Jerusalem, Israel; ${ }^{96}$ Meir Medical Center, Kfar Saba, Israel; ${ }^{97}$ The Western Galilee Hospital, Nahariya, Israel; ${ }^{98}$ Nazareth Towers Medical Center, Nazareth, Israel; ${ }^{99}$ Laniado Hospital, Netanya, Israel; ${ }^{100}$ Rabin Medical Center, Petah Tikva, Israel; ${ }^{101}$ Baruch Padeh Medical Center Poriya, Tiberias, Israel; ${ }^{102}$ Kaplan Medical Center, Rehovot, Israel; ${ }^{103}$ Ziv Medical Center, Safed, Israel; ${ }^{104} \mathrm{Tel}$ Aviv Sourasky Medical Centre, Tel Aviv, Israel; ${ }^{105}$ IRCCS San Matteo Hospital Foundation, Pavia, Italy; ${ }^{106}$ University Cattolica del Sacro Cuore, Policlinico Gemelli, Rome, Italy; ${ }^{107}$ VU University Medical Center, Amsterdam, The Netherlands; ${ }^{108}$ Rijnstate Hospital, Arnhem, The Netherlands; ${ }^{109}$ Jeroen Bosch Hospital, Den Bosch, The Netherlands; ${ }^{110}$ Slingeland Hospital, Doetinchem, The Netherlands; ${ }^{111}$ Gelderse Vallei Hospital, Ede, The Netherlands; ${ }^{112}$ Elkerliek Hospital, Helmond, The Netherlands; ${ }^{113}$ Radboudumc, Nijmegen, The Netherlands; ${ }^{114}$ Bernhoven Hospital, Uden, The Netherlands; ${ }^{115}$ Maxima Medical Center, Veldhoven, The Netherlands; ${ }^{116}$ Warszawski Uniwersytet Medyczny, Warsaw, Poland; ${ }^{117}$ Centro Hospitalar de Lisboa, Lisbon, Portugal; ${ }^{118}$ Districtual Hospital, Brasov, Romania; ${ }^{119}$ Coltea Clinical Hospital, Bucharest, Romania; ${ }^{120}$ Fundeni Clinical Institute, Bucharest, Romania; ${ }^{121}$ Clinical Center of Vojvodina, Novi Sad, Serbia; ${ }^{122}$ Hospital del Mar, Barcelona, Spain; ${ }^{123}$ Hospital Universitari Germans Trias i Pujol, Barcelona, Spain; ${ }^{124}$ Hospital Del Sas, Jerez De La Frontera, Cadiz, Spain; ${ }^{125}$ Hospital Universitario Puerta del Mar, Cadiz, Spain; ${ }^{126}$ Institute de Investigacion Biomedica, Lleida, Spain; ${ }^{127}$ Hospital Clinico Universitario San Carlos, Madrid, Spain; ${ }^{128}$ Hospital Universitario Meseguer, Murcia, Spain; ${ }^{129}$ Hospital Universitario Virgen de la Arrixaca, Murcia, Spain; ${ }^{130}$ Hospital Universitario Central de Asturias, Oviedo, Spain; ${ }^{131}$ Hospital Universitario de Salamanca, Salamaca, Spain; ${ }^{132}$ Hospital Clinico Universitario de Valencia, Valencia, Spain; ${ }^{133}$ Hospital Dr. Peset, Valencia, Spain; ${ }^{134}$ Hospital Universitario La Fe, Valencia, Spain; ${ }^{135}$ Malarsjukhuset, Eskilstuna, Sweden; ${ }^{136}$ Sahlgrenska University Hospital, Göteborg, Sweden; ${ }^{137}$ Teaching Hospital of Halmstad, Halmstad, Sweden; ${ }^{138}$ University Hospital Linköping, Linköping, Sweden; ${ }^{139}$ Sunderby Hospital, Lulea, Sweden; ${ }^{140}$ Lund University Hospital, Lund, Sweden; ${ }^{141}$ Orebro University Hospital, Orebro, Sweden; ${ }^{142}$ Karolinska University Hospital, Stockholm, Sweden; ${ }^{143}$ Södersjukhuset, Stockholm, Sweden; ${ }^{144}$ Sundsvalls sjukhus, Sundsvall, Sweden; ${ }^{145}$ Umea Regional Hospital, Umea, Sweden; ${ }^{146}$ Uppsala University, Uppsala, Sweden; ${ }^{147}$ Aberdeen Royal 
Infirmary, Aberdeen, UK; ${ }^{148}$ Queen Elizabeth Hospital, Birmingham, UK; ${ }^{149}$ Blackpool Victoria Hospital, Blackpool, UK; ${ }^{150}$ Royal Bournemouth Hospital, Bournemouth, UK; ${ }^{151}$ Bradford Royal Infirmary, Bradford, UK; ${ }^{152}$ Addenbrooke's Hospital, Cambridge, UK; ${ }^{153}$ Western Infirmary, Glasgow, UK; ${ }^{154}$ Harrogate District Hospital, Harrogate, UK; ${ }^{155}$ Huddersfield Royal Infirmary, Huddersfield, UK; ${ }^{156}$ Hull and East Yorkshire Hospitals NHS Trust, Hull, UK; ${ }^{157}$ Leeds Teaching Hospitals, Leeds, UK; ${ }^{158}$ Christie Hospital, Manchester, UK; ${ }^{159}$ Royal Victoria Infirmary, Newcastle upon Tyne, UK; ${ }^{160}$ Northampton General Hospital, Northampton, UK; ${ }^{161}$ City Hospital, Nottingham, UK; ${ }^{162}$ John Radcliffe Hospitals NHS Trust, Oxford, UK; ${ }^{163}$ Airedale NHS Trust, Steeton, UK; ${ }^{164}$ Royal Cornwall Hospital, Truro, UK; ${ }^{165}$ Mid Yorkshire Hospitals, Wakefield, UK; ${ }^{166}$ Worcestershire Acute Hospitals NHS Trust, Worcester, UK; ${ }^{167}$ York Hospital, York, UK

Funding The EUMDS Registry is supported by an educational grant from Novartis Pharmacy B.V. Oncology Europe, and Amgen Limited. This work is part of the MDS-RIGHT activities, which has received funding from the European Union's Horizon 2020 Research and Innovation Programme under Grant Agreement No. 634789 MDSRIGHT - "Providing the right care to the right patient with MyeloDysplastic Syndrome at the right time." The Lifelines Biobank initiative has been made possible by subsidy from the Dutch Ministry of Health, Welfare and Sport, the Dutch Ministry of Economic Affairs, the University Medical Center Groningen (UMCG the Netherlands), University Groningen, and the Northern Provinces of the Netherlands. The authors wish to acknowledge the services of the Lifelines Cohort Study, the contributing research centers delivering data to Lifelines, and all the study participants.

Author contributions Design: MH, TB, CvM, ASm, SL, TdW; provision of patients, assembly of data: DB, DC, SK, ASy, HG, MS, SL, AT, SK, PP, OS, EH-L, JC, MvK, HW, RR, EW, DWS; statistical analysis and interpretation: $\mathrm{MH}, \mathrm{TB}, \mathrm{CvM}, \mathrm{ASm}, \mathrm{TdW}$; manuscript writing: all authors; final approval: all authors.

\section{Compliance with ethical standards}

Conflict of interest CvM: project manager of the EUMDS Registry, is funded by the EUMDS and MDS-RIGHT project budget; ASm: research funding from Novartis, Cilag-Janssen, and Boehringer Ingelheim; ASy: honoraria and consulting fees from Amgen, Celgene/ GenesisPharma, Genzyme/Sanofi, Gilead, Janssen-Cilag, Pfizer, MSD, and Novartis; HG: honoraria from Celgene, Novartis, and Alexion; SK: honoraria from Novartis, Jazz, and Celgene; EH-L: research funding from Celgene; NB: research funding from Novartis, Bristol Meyer Squibb, Pfizer, Ariad, MSD, Astellas, Xenikos, and Celgene, educational grant from Novartis, Celgene, and Janssen-Cilag; DWS: paid employee of RadboudUMC, which offers hepcidin measurements via Hepcidinanalysis.com at a fee for service basis; TdW: research funding from Amgen, Celgene, and Novartis, as project coordinator EUMDS. The other authors declare that they have no conflict of interest.

Publisher's note Springer Nature remains neutral with regard to jurisdictional claims in published maps and institutional affiliations.

Open Access This article is licensed under a Creative Commons Attribution 4.0 International License, which permits use, sharing, adaptation, distribution and reproduction in any medium or format, as long as you give appropriate credit to the original author(s) and the source, provide a link to the Creative Commons license, and indicate if changes were made. The images or other third party material in this article are included in the article's Creative Commons license, unless indicated otherwise in a credit line to the material. If material is not included in the article's Creative Commons license and your intended use is not permitted by statutory regulation or exceeds the permitted use, you will need to obtain permission directly from the copyright holder. To view a copy of this license, visit http://creativecommons. org/licenses/by/4.0/.

\section{References}

1. Cazzola M, Della Porta MG, Malcovati L. Clinical relevance of anemia and transfusion iron overload in myelodysplastic syndromes. Hematology Am Soc Hematol Educ Program. 2008;1: 166-75.

2. Malcovati L, Porta MG, Pascutto C, Invernizzi R, Boni M, Travaglino $\mathrm{E}$, et al. Prognostic factors and life expectancy in myelodysplastic syndromes classified according to WHO criteria: a basis for clinical decision making. J Clin Oncol. 2005;23:7594-603.

3. Leitch HA, Fibach E, Rachmilewitz E. Toxicity of iron overload and iron overload reduction in the setting of hematopoietic stem cell transplantation for hematologic malignancies. Crit Rev Oncol Hematol. 2017;113:156-70.

4. Shenoy N, Vallumsetla N, Rachmilewitz E, Verma A, Ginzburg Y. Impact of iron overload and potential benefit from iron chelation in low-risk myelodysplastic syndrome. Blood. 2014;124:873-81.

5. de Swart L, Reiniers C, Bagguley T, van Marrewijk C, Bowen D, Hellström-Lindberg E, et al. Labile plasma iron levels predict survival in patients with lower-risk myelodysplastic syndromes. Haematologica. 2018;103:69-79.

6. Porter JB, de Witte T, Cappellini MD, Gattermann N. New insights into transfusion-related iron toxicity: Implications for the oncologist. Crit Rev Oncol Hematol. 2016;99:261-71.

7. Ganz T, Nemeth E. Hepcidin and iron homeostasis. Biochim Biophys Acta. 2012;1823:1434-43.

8. Kautz L, Jung G, Valore EV, Rivella S, Nemeth E, Ganz T. Identification of erythroferrone as an erythroid regulator of iron metabolism. Nat Genet. 2014;46:678-84.

9. Ye ZW, Zhang J, Townsend DM, Tew KD. Oxidative stress, redox regulation and diseases of cellular differentiation. Biochim Biophys Acta. 2015;1850:1607-21.

10. Pimková K, Chrastinová L, Suttnar J, Štikarová J, Kotlín R, Čermák $\mathrm{J}$, et al. Plasma levels of aminothiols, nitrite, nitrate, and malondialdehyde in myelodysplastic syndromes in the context of clinical outcomes and as a consequence of iron overload. Oxid Med Cell Longev. 2014;2014:416028.

11. Pilo F, Angelucci E. A storm in the niche: Iron, oxidative stress and haemopoiesis. Blood Rev. 2018;32:29-35.

12. de Souza GF, Barbosa MC, Santos TE, Carvalho TM, de Freitas RM, Martins MR, et al. Increased parameters of oxidative stress and its relation to transfusion iron overload in patients with myelodysplastic syndromes. J Clin Pathol. 2013;66:996-8.

13. Santini V, Girelli D, Sanna A, Martinelli N, Duca L, Campostrini $\mathrm{N}$, et al. Hepcidin levels and their determinants in different types of myelodysplastic syndromes. PLoS ONE. 2011;6:e23109.

14. Ambaglio I, Malcovati L, Papaemmanuil E, Laarakkers CM, Della Porta MG, Gallì A, et al. Inappropriately low hepcidin levels in patients with myelodysplastic syndrome carrying a somatic mutation of SF3B1. Haematologica. 2013;98:420-3.

15. Zipperer E, Post JG, Herkert M, Kündgen A, Fox F, Haas R, et al. Serum hepcidin measured with an improved ELISA correlates with parameters of iron metabolism in patients with myelodysplastic syndrome. Ann Hematol. 2013;92:1617-23. 